\title{
7S fragment of type iv collagen as a serum marker of canine liver fibrosis
}

\author{
K. Glińska-Suchocka1, A. Orłowska² ${ }^{2}$ K. Kubiak ${ }^{1}$, J. Spużak ${ }^{1}$, M. Jankowski ${ }^{1}$ \\ ${ }^{1}$ Department of Internal Diseases with Clinic of Horses, Dogs and Cats, Faculty of Veterinary Medicine, \\ Wroclaw University of Environmental and Life Sciences, pl. Grunwaldzki 47, 50-366 Wrocław, Poland \\ ${ }^{2}$ Department of Biochemistry, Pharmacology and Toxicology, Faculty of Veterinary Medicine, \\ Wroclaw University of Environmental and Life Sciences, Norwida 31, 50-375 Wrocław, Poland
}

\begin{abstract}
The aim of this study was to assess whether the serum levels of the 7S fragment of type IV collagen may aid in diagnosing liver fibrosis in dogs. The study was carried out on 20 dogs with liver disease. Serum levels of the 7S fragment of type IV collagen were measured in all dogs. The analysis showed that healthy dogs and dogs with type 1, 2 and 3 liver fibrosis had low serum concentrations of the 7S fragment of type IV collagen compared to dogs with type 4 liver fibrosis. The study revealed that the assessment of serum levels of the 7S fragment of type IV collagen is useful in the diagnosis of advanced liver fibrosis and cirrhosis.
\end{abstract}

Key words: liver, dog, serum 7S fragment of type IV collagen, liver fibrosis

\section{Introduction}

Hepatic fibrosis is a wound-healing process in chronic liver injury, and is characterized by the activation of hepatic stellate cells (HSC) and excess production of collagen components of the extracellular matrix together with a change in the ratio between extracellular matrix and cellular components. Liver fibrosis is the process of activation of HSC, a progressive deposition of collagen components of the extracellular matrix (ECM) and a change in the ratio between extracellular matrix components and cellular components. Direct liver remodeling marker is the $7 \mathrm{~S}$ fragment of type IV collagen (PIVNP) (Tsutsumi et al. 1993, Friedman 2000). To date, the correlation between the extent of liver fibrosis and serum levels of the 7S fragment of type IV collagen (PIVNP) has not been studied in veterinary medicine.

The aim of this study was to assess the usefulness of measuring serum levels of the 7S fragment of type IV collagen in the diagnosis of liver fibrosis in dogs.

\section{Materials and Methods}

The study was carried out on 20 dogs of different breeds and sex, aged 7 to 15 years, with suspicion of chronic liver disease. In all the dogs the blood tests (AST, ALT, ALP, GGT, total bilirubin) and liver biopsy was performed. Serum PIVNP concentrations were measured in duplicate using the Human N-terminal procollagen IV propeptide Elisa Kit

Correspondence to: K. Glińska-Suchocka, e-mail: kamila.glinska@up.wroc.pl 
(MyBioSource, San Diego, USA). A core Tru-Cut needle biopsy of the liver was carried out in all animals. The biopsies were performed under general anesthesia (xylazine $1 \mathrm{mg} / \mathrm{kg}$ i.m., atropine $0.05 \mathrm{mg} / \mathrm{kg}$ i.m. and propofol $2-6 \mathrm{mg} / \mathrm{kg}$ ). The liver biopsy was carried out under ultrasound guidance using a semi-automatic Tru - Cut needle (20 cm long, 16G). Consequently, a left-lobe and right-lobe biopsy was obtained from each dog. Two biopsy specimens (approximately $1.5 \mathrm{~cm}$ long) were obtained from the lesions.

The degree of liver fibrosis was determined using the hematoxylin and eosin, Van Gieson and Mallory's trichrome staining. Four to five portal triads were evaluated in each sample. The Scheuer scale was used to grade liver fibrosis: F0 - no fibrosis, single collagen fibres in the portal spaces; F1 - fibrosis within portal spaces with an extension of the portal tracts; F2 - peri-portal fibrosis and possibly single bridging fibrosis with the maintenance of a lobular structure; F3 - the presence of multiple fibre spans, architectural distortion, no regenerative response; F4 - diffuse fibrosis or cirrhosis (Goodman 2007).

The adoption of this classification enabled us to assess the concentration of PIVNP with respect to the severity of the lesions present in the liver tissue. Based on the degree of liver fibrosis, the dogs were divided into five groups: group 1 consisted of 5 dogs with no liver fibrosis (F0), group 2 consisted of 3 dogs with stage 1 liver fibrosis (F1), group 3 included 4 dogs with stage 2 liver fibrosis (F2), group 4 comprised 3 dogs with stage 3 liver fibrosis (F3), group 5 contained 5 dogs with stage 4 liver fibrosis (F4).

Statistical analyses were performed using STATISTICA 10 software (StatSoft Inc., Poland). Statistically significant differences between PIVNP and the level of fibrosis were determined using the Student- $t$ test. A p-value of $\leq 0.05$ was considered statistically significant.

\section{Results and Discussion}

The mean PIVNP concentration was $9.96 \pm 6.71$ $\mathrm{ng} / \mathrm{ml}$. In dogs in group $1,8.43 \pm 3.72 \mathrm{ng} / \mathrm{ml}$ in dogs in group 2, $8.37 \pm 5.70 \mathrm{ng} / \mathrm{ml}$ in group $3,13.03 \pm 3.45$ $\mathrm{ng} / \mathrm{ml}$ in group 4 and $29.1 \pm 18.98 \mathrm{ng} / \mathrm{ml}$ in group 5 . Statistically significant differences in the concentration of PIVNP were found between group 5 and group $1(\mathrm{p}=0.033)$, group 5 and group $3(\mathrm{p}=0.04)$ and group 5 and group $4(\mathrm{p}=0.032)$.

In human hepatology, collagen IV is considered to be a valuable marker of liver fibrosis, because its levels correlate with the degree of damage to the liver cells (Friedman 2000). Numerous studies evaluating the usefulness of type IV collagen in the diagnosis of liver disease have been conducted in humans (Yamada et al. 1992, Tsutsumi et al. 1993). In 1993, Tsutsumi at al. carried out a study in patients with alcoholic liver disease. Based on their results, the authors found type IV collagen to be a sensitive marker of alcoholic liver disease. These authors also found that the concentrations of type IV collagen increased in patients with hepatocellular carcinoma. In recent years, numerous studies have been conducted in human patients focusing on the use of non-invasive markers at different stages of liver fibrosis in order to determine their diagnostic potential. Yamada at al (1992) evaluated the concentrations of 7S collagen in patients with chronic hepatitis. They found those concentrations to be much higher in patients with advanced fibrosis than those with moderate fibrosis. Some authors suggest that increased serum levels of $7 \mathrm{~S}$ collagen may be associated with an enhanced metabolism in chronic liver disease (Suou et al. 1996). Numerous research groups have conducted studies on the usefulness of assessing the serum levels of PIVNP in the diagnosis of liver fibrosis. In all cases, the results indicated high diagnostic sensitivity of this marker. PIVNP was subsequently included in the liver fibrosis marker panel. To the authors' best knowledge, the correlation between the serum levels of PIVNP and the stage of liver fibrosis has not been assessed in veterinary medicine. Our research demonstrated that there is an increase in the serum concentration of PIVNP in dogs with advanced liver fibrosis (stage 4 - group 5). These results allow dogs with advanced liver fibrosis (group 5) to be differentiated from those with moderate liver fibrosis. However, there were no statistically significant differences in the serum levels of PIVNP between groups 1, 2, 3 and 4. This signifies that PIVNP cannot be used to assess the progression of liver fibrosis. Interestingly, we found that the mean serum concentration of PIVNP $(29.1 \pm 18.98 \mathrm{ng} / \mathrm{ml})$ in dogs with liver cirrhosis was greater than the mean PIVNP values obtained in human patients with liver cirrhosis $(6.9 \pm 3.5 \mathrm{ng} / \mathrm{ml})$ (Park et al. 2003).

We have found that the evaluation of serum PIVNP levels is useful in the diagnosis of advanced liver fibrosis (liver cirrhosis). However, this marker should be interpreted in association with other non-invasive markers of liver fibrosis to determine the stage of liver fibrosis.

\section{References}

Friedman SL (2000) Molecular regulation of hepatic fibrosis, an integrated cellular response to tissue injury. J Biol Chem 275: 2247-2250. 
Goodman ZD (2007) Grading and staging systems for inflammation and fibrosis in chronic liver diseases. J Hepatol 47: 598-607.

Park JH, Park CK, Kim ES, Park SY, Jo CM, Tak WY, Kweon YO, Kim SK, Choi YW (2003) The diagnostic value of serum hyaluronic acid, 7S domain of type IV collagen and AST/ALT ratio as markers of hepatic fibrosis in chronic hepatitis B and cirrhosis patients. Taehan Kan Hakhoe Chi 9: 79-88.

Suou T, Yamada S, Hosho K, Yoshikawa N, Kawasaki $\mathrm{H}$ (1996) Relationship between serum and hepatic $7 \mathrm{~S}$ fragments of IV collagen in chronic liver disease. Hepatology 23: 1154-1158.

Tsutsumi M, Urashima S, Matsuda Y, Takase S, Takada A (1993) Changes in type IV collagen content in livers of patients with alcoholic liver disease. Hepatology 17: 820-827.

Yamada S, Kishimoto Y, Suou T, Kawasaki H, Horie $\mathrm{Y}$ (1992) Heterogeneity of antigens related to $7 \mathrm{~S}$ domain of type IV collagen in serum: application to patients with chronic liver disease. Clin Chim Acta 212: 73-78. 